\title{
LEXenstein: A Framework for Lexical Simplification
}

\author{
Gustavo Henrique Paetzold and Lucia Specia \\ Department of Computer Science \\ University of Sheffield, UK \\ \{ghpaetzold1, 1.specia\}@sheffield.ac.uk
}

\begin{abstract}
Lexical Simplification consists in replacing complex words in a text with simpler alternatives. We introduce LEXenstein, the first open source framework for Lexical Simplification. It covers all major stages of the process and allows for easy benchmarking of various approaches. We test the tool's performance and report comparisons on different datasets against the state of the art approaches. The results show that combining the novel Substitution Selection and Substitution Ranking approaches introduced in LEXenstein is the most effective approach to Lexical Simplification.
\end{abstract}

\section{Introduction}

The goal of a Lexical Simplification (LS) approach is to replace complex words and expressions in a given text, often a sentence, with simpler alternatives of equivalent meaning in context. Although very intuitive, this is a challenging task since the substitutions must preserve both the original meaning and the grammaticality of the sentence being simplified.

The LS task has been gaining significant attention since the late 1990's, thanks to the positive influence of the early work presented by (Devlin and Tait, 1998) and (Carroll et al., 1999). More recently, the LS task at SemEval-2012 (Specia et al., 2012) has given LS wider visibility. Participants had the opportunity to compare their approaches in the task of ranking candidate substitutions, all of which were already known to fit the context, according to their "simplicity".

Despite its growth in popularity, the inexistence of tools to support the process and help researchers to build upon has been hampering progress in the area. We were only able to find one tool for LS: a set of scripts designed for the training and testing of ranking models provided by (Jauhar and Specia, 2012) ${ }^{1}$. However, they cover only one step of the process. In an effort to tackle this issue, we present LEXenstein: a framework for Lexical Simplification development and benchmarking.

LEXenstein is an easy-to-use framework that provides simplified access to many approaches for several sub-tasks of the LS pipeline, which is illustrated in Figure 1. Its current version includes methods for the three main sub-tasks in the pipeline: Substitution Generation, Substitution Selection and Substitution Ranking.

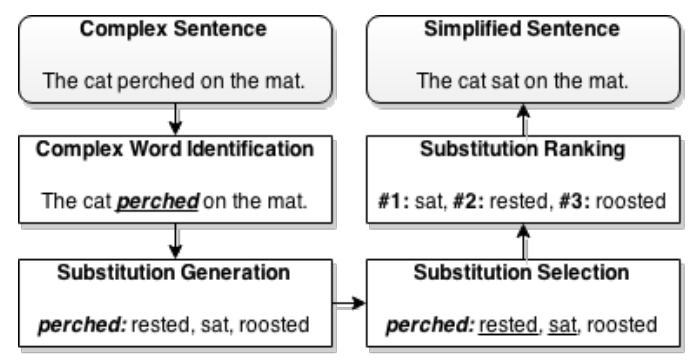

Figure 1: Lexical Simplification Pipeline

LEXenstein was devised to facilitate performance comparisons among various LS approaches, as well as the creation of new strategies for LS. In the following Sections we present LEXenstein's components (Section 2) and discuss the results of several experiments conducted with the tool (Section 3).

\section{System Overview}

LEXenstein is a Python library that provides several approaches for sub-tasks in LS. To increase its flexibility, the library is structured in six modules: Substitution Generation, Substitution Selection, Substitution Ranking, Feature Estimation, Evaluation and Text Adorning. In the following Sections, we describe them in more detail.

\footnotetext{
${ }^{1}$ https://github.com/sjauhar/simplex
} 


\subsection{Substitution Generation}

We define Substitution Generation (SG) as the task of producing candidate substitutions for complex words, which is normally done regardless of the context of the complex word. Previous work commonly addresses this task by querying general domain thesauri such as WordNet (Fellbaum, 1998), or domain specific ones such as UMLS (Bodenreider, 2004). Examples of work resorting to this strategy are (Devlin and Tait, 1998) and (Carroll et al., 1999). Recent work focuses on learning substitutions from sentence-aligned parallel corpora of complex-simple texts (Paetzold and Specia, 2013; Horn et al., 2014).

LEXenstein's SG module offers support for five approaches. All approaches use LEXenstein's Text Adorning module to create substitutions for all possible inflections of verbs and nouns. Each approach is represented by one of the following Python classes:

KauchakGenerator (Horn et al., 2014) Automatically extracts substitutions from parallel corpora. It requires a set of tagged parallel sentences and the word alignments between them in Pharaoh format (Och and Ney, 2000). It produces a dictionary of complex-to-simple substitutions filtered by the criteria described in (Horn et al., 2014).

BiranGenerator (Biran et al., 2011) Filters substitutions based on the Cartesian product between vocabularies of complex and simple words. It requires vocabularies of complex and simple words, as well as two language models trained over complex and simple corpora. It produces a dictionary linking words to a set of synonyms and hypernyms filtered by the criteria described in (Biran et al., 2011).

YamamotoGenerator (Kajiwara et al., 2013) Extracts substitutions from dictionary definitions of complex words. It requires an API key for the Merriam Dictionary ${ }^{2}$, which can be obtained for free. It produces a dictionary linking words in the Merriam Dictionary and WordNet to words with the same Part-of-Speech (POS) tag in its entries' definitions and examples of usage.

MerriamGenerator Extracts a dictionary linking words to their synonyms, as listed in the Merriam Thesaurus. It requires an API key.

\footnotetext{
${ }^{2}$ http://www.dictionaryapi.com/
}

WordnetGenerator Extracts a dictionary linking words to their synonyms, as listed in WordNet.

\subsection{Substitution Selection}

Substitution Selection (SS) is the task of selecting which substitutions - from a given list - can replace a complex word in a given sentence without altering its meaning. Most work addresses this task referring to the context of the complex word by employing Word Sense Disambiguation (WSD) approaches (Sedding and Kazakov, 2004; Nunes et al., 2013), or by discarding substitutions which do not share the same POS tag of the target complex word (Kajiwara et al., 2013; Paetzold and Specia, 2013).

LEXenstein's SS module provides access to three approaches. All approaches require as input a dictionary of substitutions generated by a given approach and a dataset in the VICTOR format (as in Victor Frankenstein (Shelley, 2007)). As output, they produce a set of selected substitutions for each entry in the VICTOR dataset. The VICTOR format is structured as illustrated in Example 1, where $S_{i}$ is the $i$ th sentence in the dataset, $w_{i}$ a target complex word in the $h_{i}$ th position of $S_{i}, c_{i}^{j}$ a substitution candidate and $r_{i}^{j}$ its simplicity ranking. Each bracketed component is separated by a tabulation marker.

$$
\left[\begin{array}{cc}
\left\langle\mathrm{S}_{1}\right\rangle\left\langle\mathrm{w}_{1}\right\rangle\left\langle\mathrm{h}_{1}\right\rangle\left\langle\mathrm{r}_{1}^{1}: \mathrm{c}_{1}^{1}\right\rangle \cdots\left\langle\mathrm{r}_{1}^{n}: \mathrm{c}_{1}^{n}\right\rangle \\
\vdots \\
\left\langle\mathrm{S}_{m}\right\rangle\left\langle\mathrm{w}_{m}\right\rangle\left\langle\mathrm{h}_{m}\right\rangle\left\langle\mathrm{r}_{m}^{1}: \mathrm{c}_{m}^{1}\right\rangle \cdots\left\langle\mathrm{r}_{m}^{n}: \mathrm{c}_{m}^{n}\right\rangle
\end{array}\right]
$$

LEXenstein includes two resources for training/testing in the VICTOR format: the LexMTurk (Horn et al., 2014) and the SemEval corpus (Specia et al., 2012). Each approach in the SS module is represented by one of the following Python classes:

WSDSelector Allows for the user to use one among various classic WSD approaches in SS. It requires the PyWSD (Tan, 2014) module to be installed, which includes the approaches presented by (Lesk, 1986) and (Wu and Palmer, 1994), as well as baselines such as random and first senses.

BiranSelector (Biran et al., 2011) Employs a strategy in which a word co-occurrence model is used to determine which substitutions have meaning similar to that of a target complex word. It requires a plain text file with each line in the format specified in Example 2, where $\left\langle w_{i}\right\rangle$ is a word, 
$\left\langle c_{i}^{j}\right\rangle$ a co-occurring word and $\left\langle f_{i}^{j}\right\rangle$ its frequency of occurrence.

$$
\left\langle w_{i}\right\rangle\left\langle c_{i}^{0}\right\rangle:\left\langle f_{i}^{0}\right\rangle \cdots\left\langle c_{i}^{n}\right\rangle:\left\langle f_{i}^{n}\right\rangle
$$

Each component in the format in 2 must be separated by a tabulation marker. Given such a model, the approach filters all substitutions which are estimated to be more complex than the target word, and also those for which the distance between their co-occurrence vector and the target sentence's vector is higher than a threshold set by the user.

WordVectorSelector Employs a novel strategy, in which a word vector model is used to determine which substitutions have the closest meaning to that of the sentence being simplified. It requires a binary word vector model produced by Word $2 \mathrm{Vec}^{3}$, and can be configured in many ways. It retrieves a user-defined percentage of the substitutions, which are ranked with respect to the cosine distance between their word vector and the sum of some or all of the sentences' words, depending on the settings defined by the user.

\subsection{Substitution Ranking}

Substitution Ranking (SR) is the task of ranking a set of selected substitutions for a target complex word with respect to their simplicity. Approaches vary from simple word length and frequencybased measures (Devlin and Tait, 1998; Carroll et al., 1998; Carroll et al., 1999; Biran et al., 2011) to more sophisticated linear combinations of scoring functions (Jauhar and Specia, 2012), as well as machine learning-based approaches (Horn et al., 2014).

LEXenstein's SR module provides access to three approaches. All approaches receive as input datasets in the VICTOR format, which can be either training/testing datasets already containing only valid substitutions in context, or datasets generated with (potentially noisy) substitutions by a given SS approach. They also require as input a FeatureEstimator object to calculate feature values describing the candidate substitutes. More details on the FeatureEstimator class are provided in Section 2.4. Each approach in the SR module is represented by one of the following Python classes:

\footnotetext{
${ }^{3}$ https://code.google.com/p/word2vec/
}

MetricRanker Employs a simple ranking strategy based on the values of a single feature provided by the user. By configuring the input FeatureEstimator object, the user can calculate values of several features for the candidates in a given dataset and easily rank the candidates according to each of these features.

SVMRanker (Joachims, 2002) Use Support Vector Machines in a setup that minimises a loss function with respect to a ranking model. This strategy is the one employed in the LS experiments of (Horn et al., 2014), yielding promising results. The user needs to provide a path to their SVMRank installation, as well as SVM-related configurations, such as the kernel type and parameter values for $C$, epsilon, etc.

BoundaryRanker Employs a novel strategy, in which ranking is framed as a binary classification task. During training, this approach assigns the label 1 to all candidates of rank $1 \geq r \geq p$, where $p$ is a range set by the user, and 0 to the remaining candidates. It then trains a stochastic descent linear classifier based on the features specified in the FeatureEstimator object. During testing, candidate substitutions are ranked based on how far from 0 they are. This ranker allows the user to provide several parameters during training, such as loss function and penalty type.

\subsection{Feature Estimation}

LEXenstein's Feature Estimation module allows the calculation of several features for LS-related tasks. Its class FeatureEstimator allows the user to select and configure many features commonly used by LS approaches.

The FeatureEstimator object can be used either for the creation of LEXenstein's rankers, or in stand-alone setups. For the latter, the class provides a function called calculateFeatures, which produces a matrix $M \mathrm{x} N$ containing $M$ feature values for each of the $N$ substitution candidates listed in the dataset. Each of the 11 features supported must be configured individually. They can be grouped in four categories:

Lexicon-oriented: Binary features which receive value 1 if a candidate appears in a given vocabulary, and 0 otherwise.

Morphological: Features that exploit morphological characteristics of substitutions, such as word length and number of syllables. 
Collocational: N-gram probabilities of the form $P\left(S_{h-1}^{h-l} c S_{h+1}^{h+r}\right)$, where $c$ is a candidate substitution in the $h$ th position in sentence $S$, and $S_{h-1}^{h-l}$ and $S_{h+1}^{h+r}$ are n-grams of size $l$ and $r$, respectively.

Sense-oriented: Several features which are related to the meaning of a candidate substitution such as number of senses, lemmas, synonyms, hypernyms, hyponyms and maximum and minimum distances among all of its senses.

\subsection{Evaluation}

Since one of the goals of LEXenstein is to facilitate the benchmarking LS approaches, it is crucial that it provides evaluation methods. This module includes functions for the evaluation of all subtasks, both individually and in combination. It contains four Python classes:

GeneratorEvaluator: Provides evaluation metrics for SG methods. It requires a gold-standard in the VICTOR format and a set of generated substitutions. It returns the Potential, Precision and F-measure, where Potential is the proportion of instances for which at least one of the substitutions generated is present in the gold-standard, Precision the proportion of generated instances which are present in the gold-standard, and F-measure their harmonic mean.

SelectorEvaluator: Provides evaluation metrics for SS methods. It requires a gold-standard in the VICTOR format and a set of selected substitutions. It returns the Potential, Precision and Fmeasure of the SS approach, as defined above.

RankerEvaluator: Provides evaluation metrics for SR methods. It requires a gold-standard in the VICTOR format and a set of ranked substitutions. It returns the TRank-at-1:3 and Recall-at-1:3 metrics (Specia et al., 2012), where Trank-at- $i$ is the proportion of instances for which a candidate of gold-rank $r \leq i$ was ranked first, and Recall-at- $i$ the proportion of candidates of gold-rank $r \leq i$ that are ranked in positions $p \leq i$.

PipelineEvaluator: Provides evaluation metrics for the entire LS pipeline. It requires as input a gold-standard in the VICTOR format and a set of ranked substitutions which have been generated and selected by a given set of approaches. It returns the approaches' Precision, Accuracy and
Change Proportion, where Precision is the proportion of instances for which the highest ranking substitution is not the target complex word itself and is in the gold-standard, Accuracy is the proportion of instances for which the highest ranking substitution is in the gold-standard, and Change Proportion is the proportion of instances for which the highest ranking substitution is not the target complex word itself.

\subsection{Text Adorning}

This approach provides a Python interface to the Morph Adorner Toolkit (Paetzold, 2015), a set of Java tools that facilitates the access to Morph Adorner's functionalities. The class provides easy access to word lemmatisation, word stemming, syllable splitting, noun inflection, verb tensing and verb conjugation.

\subsection{Resources}

LEXenstein also provides a wide array of resources for the user to explore in benchmarking tasks. Among them are the aforementioned LexMturk and SemEval corpora in the VICTOR format, lists of stop and basic words, as well as language models and lexica built over Wikipedia and Simple Wikipedia.

\section{Experiments}

In this Section, we discuss the results obtained in four benchmarking experiments.

\subsection{Substitution Generation}

In this experiment we evaluate all SG approaches in LEXenstein. For the KauchakGenerator, we use the corpus provided by (Kauchak, 2013), composed of 150, 569 complex-to-simple parallel sentences, parsed by the Stanford Parser (Klein and Manning, 1965). From the the same corpus, we build the required vocabularies and language models for the BiranGenerator. We used the LexMturk dataset as the gold-standard (Horn et al., 2014), which is composed by 500 sentences, each with a single target complex word and 50 substitutions suggested by turkers. The results are presented in Table 1.

The results in Table 1 show that the method of (Horn et al., 2014) yields the best F-Measure results, although combining the output of all generation methods yields the highest Potential. This shows that using parallel corpora to generate substitution candidates for complex words can be a 


\begin{tabular}{lccc}
\hline Approach & Pot. & Prec. & F \\
\hline Kauchak & 0.830 & $\mathbf{0 . 1 5 5}$ & $\mathbf{0 . 2 6 2}$ \\
Wordnet & 0.608 & 0.109 & 0.184 \\
Biran & 0.630 & 0.102 & 0.175 \\
Merriam & 0.540 & 0.067 & 0.120 \\
Yamamoto & 0.504 & 0.054 & 0.098 \\
\hline All & $\mathbf{0 . 9 7 6}$ & 0.066 & 0.124 \\
\hline
\end{tabular}

Table 1: SG benchmarking results

more efficient strategy than querying dictionaries and databases. We must, however, keep in mind that the sentences that compose the LexMturk corpus were extracted from Wikipedia, which is the same corpus from which the KauchakGenerator learns substitutions.

\subsection{Substitution Selection}

Here we evaluate of all SS approaches in LEXenstein. For the BiranSelector, we trained a cooccurrence model over a corpus of $6+$ billion words extracted from the various sources suggested in the Word2Vec documentation ${ }^{4}$, the same sources over which the word vector model required by the WordVectorSelector was trained. In order to summarise the results, we present the scores obtained only with the best performing configurations of each approach. The LexMturk corpus is used as the gold-standard, and the initial set of substitutions is the one produced by all SG approaches combined. The results are presented in Table 2.

\begin{tabular}{lcccc}
\hline Approach & Pot. & Prec. & F & Size \\
\hline Word Vec. & 0.768 & $\mathbf{0 . 2 1 9}$ & $\mathbf{0 . 3 4 1}$ & 3,042 \\
Biran & 0.508 & 0.078 & 0.136 & 9,680 \\
First & 0.176 & 0.045 & 0.072 & 2,471 \\
Lesk & 0.246 & 0.041 & 0.070 & 4,716 \\
Random & 0.082 & 0.023 & 0.035 & 2,046 \\
Wu-Pa & 0.038 & 0.013 & 0.020 & 1,749 \\
\hline No Sel. & $\mathbf{0 . 9 7 6}$ & 0.066 & 0.124 & 26,516 \\
\hline
\end{tabular}

Table 2: SS benchmarking results

"Size" in Table 2 represents the total number of substitutions selected for all test instances. The results in Table 2 show that our novel word vector approach outperforms all others in F-Measure by a considerable margin, including the method of not performing selection at all. Note that not performing selection allows for Potential to be higher, but

\footnotetext{
${ }^{4}$ https://code.google.com/p/word2vec/
}

yields very poor Precision.

\subsection{Substitution Ranking}

In Table 3 we present the results of the evaluation of several SR approaches. We trained the SVMRanker with features similar to the ones used in (Horn et al., 2014), and the BoundaryRanker with a set of 10 features selected through univariate feature selection. We compare these approaches to three baseline Metric Rankers, which use the word's frequency in Simple Wikipedia, its length or its number of senses. The SemEval corpus is used as the gold-standard so that we can compare our results with the best one obtained at SemEval2012 (Jauhar and Specia, 2012) (SemEval, in Table 3).

\begin{tabular}{lcccc}
\hline Approach & TR-1 & Rec-1 & Rec-2 & Rec-3 \\
\hline Boundary & $\mathbf{0 . 6 5 5}$ & $\mathbf{0 . 6 0 8}$ & 0.602 & 0.663 \\
SVM & 0.486 & 0.451 & 0.502 & 0.592 \\
Freq. & 0.226 & 0.220 & 0.236 & 0.300 \\
Length & 0.180 & 0.175 & 0.200 & 0.261 \\
Senses & 0.130 & 0.126 & 0.161 & 0.223 \\
\hline SemEval & 0.602 & 0.575 & $\mathbf{0 . 6 8 9}$ & $\mathbf{0 . 7 6 9}$ \\
\hline
\end{tabular}

Table 3: SR benchmarking results

The novel Boundary ranking approach outperforms all other approaches in both TRank-at-1 and Recall-at- 1 by a considerable margin, but it is worse than the best SemEval-2012 approach in terms of Recall-at-2 and 3. This however reveals not a limitation but a strength of our approach: since the Boundary ranker focuses on placing the best substitution in the highest rank, it becomes more effective at doing so as opposed to at producing a full ranking for all candidates.

\subsection{Round-Trip Evaluation}

In this experiment we evaluate the performance of different combinations of SS and SR approaches in selecting suitable substitutions for complex words from the ones produced by all generators combined. Rankers and selectors are configured in the same way as they were in the experiments in Sections 3.3 and 3.2. The gold-standard used is LexMturk, and the performance metric used is the combination's Precision: the proportion of times in which the candidate ranked highest is not the target complex word itself and belongs to the goldstandard list. Results are shown in Table 4.

The results show that combining the WordVectorSelector with the BoundaryRanker yields 


\begin{tabular}{lccc}
\hline & No Sel. & Word Vector & Biran \\
\hline Boundary & 0.342 & $\mathbf{0 . 5 5 0}$ & 0.197 \\
SVM & 0.108 & 0.219 & 0.003 \\
Freq. & 0.114 & 0.501 & 0.096 \\
Length & 0.120 & 0.408 & 0.092 \\
Senses & 0.214 & 0.448 & 0.122 \\
\hline
\end{tabular}

Table 4: Round-trip benchmarking results

the highest performance in the pipeline evaluation. Interestingly, the SVMRanker, which performed very well in the individual evaluation of Section 3.3, was outperformed by all three baselines in this experiment.

\section{Final Remarks}

We have presented LEXenstein, a framework for Lexical Simplification distributed under the permissive BSD license. It provides a wide arrange of useful resources and tools for the task, such as feature estimators, text adorners, and various approaches for Substitution Generation, Selection and Ranking. These include methods from previous work, as well as novel approaches. LEXenstein's modular structure also allows for one to easily add new approaches to it.

We have conducted evaluation experiments including various LS approaches in the literature. Our results show that the novel approaches introduced in this paper outperform those from previous work. In the future, we intend to incorporate in LEXenstein approaches for Complex Word Identification, as well as more approaches for the remaining tasks of the usual LS pipeline.

The tool can be downloaded from: http:// ghpaetzold.github.io/LEXenstein/.

\section{References}

O. Biran, S. Brody, and N. Elhadad. 2011. Putting it Simply: a Context-Aware Approach to Lexical Simplification. The 49th Annual Meeting of the ACL.

O. Bodenreider. 2004. The unified medical language system (umls): integrating biomedical terminology. Nucleic acids research.

J. Carroll, G. Minnen, Y. Canning, S. Devlin, and J. Tait. 1998. Practical simplification of english newspaper text to assist aphasic readers. In The 15th AAAI.

J. Carroll, G. Minnen, D. Pearce, Y. Canning, S. Devlin, and J. Tait. 1999. Simplifying Text for Language Impaired Readers. The 9th EACL.
S. Devlin and J. Tait. 1998. The use of a psycholinguistic database in the simplification of text for aphasic readers. Linguistic Databases.

C. Fellbaum. 1998. WordNet: An Electronic Lexical Database. Bradford Books.

C. Horn, C. Manduca, and D. Kauchak. 2014. Learning a Lexical Simplifier Using Wikipedia. The 52nd Annual Meeting of the ACL.

S.K. Jauhar and L. Specia. 2012. UOW-SHEF: SimpLex-lexical simplicity ranking based on contextual and psycholinguistic features. The 1st *SEM.

T. Joachims. 2002. Optimizing search engines using clickthrough data. In The 8th ACM.

T. Kajiwara, H. Matsumoto, and K. Yamamoto. 2013. Selecting Proper Lexical Paraphrase for Children.

D. Kauchak. 2013. Improving Text Simplification Language Modeling Using Unsimplified Text Data. The 51st Annual Meeting of the ACL.

D. Klein and C.D. Manning. 1965. Accurate Unlexicalized Parsing. In The 41st Annual Meeting of ACL.

M. Lesk. 1986. Automatic sense disambiguation using machine readable dictionaries: how to tell a pine cone from an ice cream cone. In The 5th SIGDOC.

B.P. Nunes, R. Kawase, P. Siehndel, M.A. Casanova, and S. Dietze. 2013. As Simple as It Gets - A Sentence Simplifier for Different Learning Levels and Contexts. IEEE 13th ICALT.

F.J. Och and H. Ney. 2000. Improved statistical alignment models. In The 38th Annual Meeting of the $A C L$.

G.H. Paetzold and L. Specia. 2013. Text simplification as tree transduction. In The 9th STIL.

G.H. Paetzold. 2015. Morph adorner toolkit: Morph adorner made simple. http:/ghpaetzold.github.io/MorphAdornerToolkit/.

J. Sedding and D. Kazakov. 2004. Wordnet-based text document clustering. In The 3rd ROMAND.

M. Shelley. 2007. Frankenstein. Pearson Education.

L. Specia, S.K. Jauhar, and R. Mihalcea. 2012. Semeval-2012 task 1: English lexical simplification. In The 1st *SEM.

L. Tan. 2014. Pywsd: Python implementations of word sense disambiguation technologies. https://github.com/alvations/pywsd.

Z. Wu and M. Palmer. 1994. Verbs semantics and lexical selection. In The 32nd Annual Meeting of ACL. 